\title{
An Optical Impedance Investigation of a Gold Electrodeposition System
}

\author{
Benedetto Bozzini*, Claudio Mele and Lucia D'Urzo \\ Dipartimento di Ingegneria dell'Innovazione, Università del Salento (formerly: Università di Lecce), via Monteroni, \\ I-73100 Lecce, Italy
}

\begin{abstract}
In this paper in situ visible electroreflectance measurements have been carried out in order to extract new electrochemical information from the dependence of $\Delta R / R$ on the frequency of potential modulation. The behaviour of some typical components of a Au electrodeposition bath has been investigated: a metal salt $\left(\mathrm{KAu}(\mathrm{CN})_{2}\right)$, a supporting electrolyte $\left(\mathrm{NaClO}_{4}\right)$, a metal cation complexing agent $(\mathrm{KCN})$. The voltammetric behaviour of the relevant systems has been investigated by means of Cyclic Voltammetry (CV) and Electrochemical Impedance Spectrometry (EIS). The optical and current density dynamics have been compared applying a potential square-wave modulation with frequencies in the range $1 \div 300 \mathrm{~Hz}$ and have been found to exhibit clearly different time-constants. Sensitivity to the electrolyte chemistry and radiation-wavelength have been proved for optical impedance.
\end{abstract}

Keywords: Electrodeposition, electroreflectance, gold, optical impedance, electrochemical impedance spectrometry.

\section{INTRODUCTION}

In situ VIS-UV spectroelectrochemical measurements especially when surface-sensitivity is required - are very often carried out in the so-called "electroreflectance" mode whence the acronym ERS (ElectroReflectance Spectroscopy) -, implying switching of the electrode between two different states and synchronised recording of the optical response [15]. From the experimental point of view, this is typically carried out by imposing a square-wave of electrodic potential and recording with a lock-in logic the signal of the detector receiving the radiation - generally at a fixed wavelength reflected off the electrodic surface [1-6]. The experimental details, regarding the choice of the square-wave frequency for optimal optical response (typically in the range $\mathrm{Hz} \div \mathrm{kHz}$ ) are hardly provided in the open literature. Nevertheless, hands-on experience with ERS measurements reveals that the choice of such optimal frequency: (i) is vital for the possibility of recording a $\Delta R / R$ signal with an adequate signalto-noise level; (ii) is extremely system-dependent (i.e. different optimal frequencies are found for different electrodeelectrolyte combinations as well as for different potential ranges); (iii) at excessively high modulation frequencies, $\Delta \mathrm{R} / \mathrm{R}$ tends to vanish.

An analytical expression for the frequency dependence of the electroreflectance for redox reaction of solution-phase species at the electrode surface was derived and experimentally verified in [7]. In [8-10] is reported that simultaneous measurements of Surface Conductance (SC), Cyclic Voltammetry (CV) and Electroreflectance can provide a sensitive method for observing the electrochemical interface. SC is compared with reflectance measurements of gold films when no faradaic currents or significant adsorption exist and also in the presence of ionic adsorption [9]. It is showed that the reflectance response is sensitive to the ionic adsorption giving information on the changes at the electrode surface. In

*Address correspondence to this author at the Dipartimento di Ingegneria dell'Innovazione, Università del Salento (formerly: Università di Lecce), via Monteroni, I-73100 Lecce, Italy; Tel: 00390832 297375;

E-mail: benedetto.bozzini@unile.it
[10] it is demonstrated that while the electroreflectance effect is primarily caused by interband transitions and the surface free electron concentration, the conductance effect is due to the average free electron density and the scattering of conduction electrons by adsorbed species. In [11] is reported a method to obtain the derivative of the voltammogram through resistance measurements, useful for several electrochemical processes occurring at the metal-solution interface. In [12] a modified version of this technique and an interpretation of the results of its practical applications are also given.

This paper means to address the problem of rationalising the choice of the potential-modulation frequency in ERS measurements, as applied to some experimental cases relevant to the electrodeposition of $\mathrm{Au}$ as well as to call attention to the possibility of extracting new electrochemical information from the dependence of $\Delta R / R$ on the frequency $\omega$ of potential modulation. In this paper we have investigated the effects of some typical components of a Au-electrodeposition bath: (i) the metal salt $\left(\mathrm{KAu}(\mathrm{CN})_{2}\right)$; (ii) the supporting electrolyte (in this particular case $\mathrm{NaClO}_{4}$ ); (iii) the metal cation complexing agent $(\mathrm{KCN})$. In order to investigate the effects of the single substances, we have studied the dynamic optical behaviour of a polycrystalline Au electrode in contact with aqueous solutions of: (i) $\mathrm{NaClO}_{4}$; (ii) $\mathrm{NaClO}_{4}$ and $\mathrm{KCN}$; (iii) $\mathrm{NaClO}_{4}$ and $\mathrm{KAu}(\mathrm{CN})_{2}$. For the three systems investigated, we assessed the potential ranges in which no faradaic reaction other than oxygen reduction take place, in order to modulate the potentials between couples of potentials at which the electrode behaviour is expected to be highly reversible. In order to compare the optical and current-density (c.d.) dynamics, we recorded both optical $\Delta \mathrm{R} / \mathrm{R}$ values and c.d. transients in the frequency range accessible to the ERS spectrometer, whose properties have been detailed in [6].

\section{EXPERIMENTAL}

We studied the following solutions: (i) $\mathrm{NaClO}_{4} 0.1 \mathrm{M}$; (ii) $\mathrm{NaClO}_{4} 0.1 \mathrm{M}, \mathrm{KCN} 25 \mathrm{mM}$; (iii) $\mathrm{NaClO}_{4} 0.1 \mathrm{M}$, $\mathrm{KAu}(\mathrm{CN})_{2} 25 \mathrm{mM}$. The solutions were prepared from analytic grade chemicals and ultra-pure water with a resistivity 
of $18.2 \mathrm{M} \Omega \mathrm{cm}$. For the sake of experimental simplicity and in order to keep the chemical conditions closer to industrially relevant ones, we did not outgas the solutions. Of course, the presence of $\mathrm{O}_{2}$ and its reduction have no sizable bearing on the Au electrodepostion process. No measurable $\mathrm{pH}$ changes occurred in the course of our experiments.

Cyclic voltammograms (CVs), Electrochemical Impedance Spectrometry (EIS) and c.d. transients corresponding to the imposition of potential square-wave modulations were recorded with an AMEL 5000 programmable potentiostat. The working electrode was a polycrystalline $\mathrm{Au}$; the electrode was annealed in a butane flame and quenched in ultrapure water before each measurement. The reference electrode was an AMEL $\mathrm{Ag} / \mathrm{AgCl}(\mathrm{KCl} 3 \mathrm{M})$; potential values are reported vs. $\mathrm{Ag} / \mathrm{AgCl}$. The reference electrode probe tip was placed at a distance of ca. $5 \mathrm{~mm}$ from the working electrode. The counter electrode was a platinised Ti expandedmesh electrode exhibiting an area of ca. $10 \mathrm{~cm}^{2}$.

ERS measurements were carried out in a cell with a vertical polycrystalline Au disc working electrode of diameter 5 $\mathrm{mm}$ embedded in a Teflon cylindrical holder. A metallographic polishing procedure, consisting of wet grinding with 2400 grit $\mathrm{SiC}$ paper, proved adequate for an excellent reproducibility of the reflectivity of the electrode at the beginning of each series of experiments. The counter electrode was a Pt wire loop $\left(1.25 \mathrm{~cm}^{2}\right)$ concentric and coplanar with the working electrode disc. The reference electrode was placed in a separate compartment, the probe tip was placed 3 $\mathrm{mm}$ from the rim of the working electrode disc. p-polarised light at an incidence angle of $45^{\circ}$ was chosen. The $\Delta R / R$ signal was detected with a lock-in technique. Solid state light sources were employed: a set of three LEDs was used to cover the VIS and UV range $(220 \div 670 \mathrm{~nm})$. The wavelength was selected with a grating monochromator with a spectral resolution of $7 \mathrm{~nm}$. A photodiode detector was employed.

\section{RESULTS AND DISCUSSION}

The three electrolytes investigated were chosen in order to gain information on different, concurrent aspects of the interfacial electrochemistry of Au plating: (i) electrodic charge density; (ii) adsorption of non-electroactive species; (iii) adsorption of species undergoing electrochemical reduction. The reasons of our choice of chemicals are detailed below. (i) We selected a non-specifically adsorbing supporting electrolyte: $\mathrm{NaClO}_{4}$; we expect that the optical behaviour of the $\mathrm{Au}$ electrode in $\mathrm{NaClO}_{4}$-containing aqueous solutions is dominated by surface-charging effects (i.e electroreflectance in the strict sense). (ii) KCN, exhibiting strong specific adsorption at $\mathrm{Au}$ electrodes, is a classic complexing agent for $\mathrm{Au}(\mathrm{I})$ and $\mathrm{Au}(\mathrm{III})$ baths, allowing the growth of Au layers of high functional and aesthetic quality. (iii) $\mathrm{KAu}(\mathrm{CN})_{2}$ is he Au-salt most commonly used in industrial systems. The study of the three electrolytes described in Section 2 allows to highlight separately some aspects of electrochemical and optical dynamic response of the chief ingredients of a typical industrial bath.

\subsection{Cyclic Voltammetry and Electrochemical Impedance Spectrometry Measurements}

In order to assess the potential regions corresponding to the relevant faradaic reactions, Cyclic Voltammetry (CV) measurements were carried out. The nature of the electrochemical processes prevailing in different frequency ranges was investigated by Electrochemical Impedance Spectrometry (EIS). In Fig. (1) we show CVs for the three systems investigated, measured with a scan rate of $50 \mathrm{mV} \mathrm{s}^{-1}$. It can be observed that a DLC (strictly so, in the absence of dissolved $\mathrm{O}_{2}$ ) region exists between ca. 0 and $400 \mathrm{mV}$ for all the systems considered.

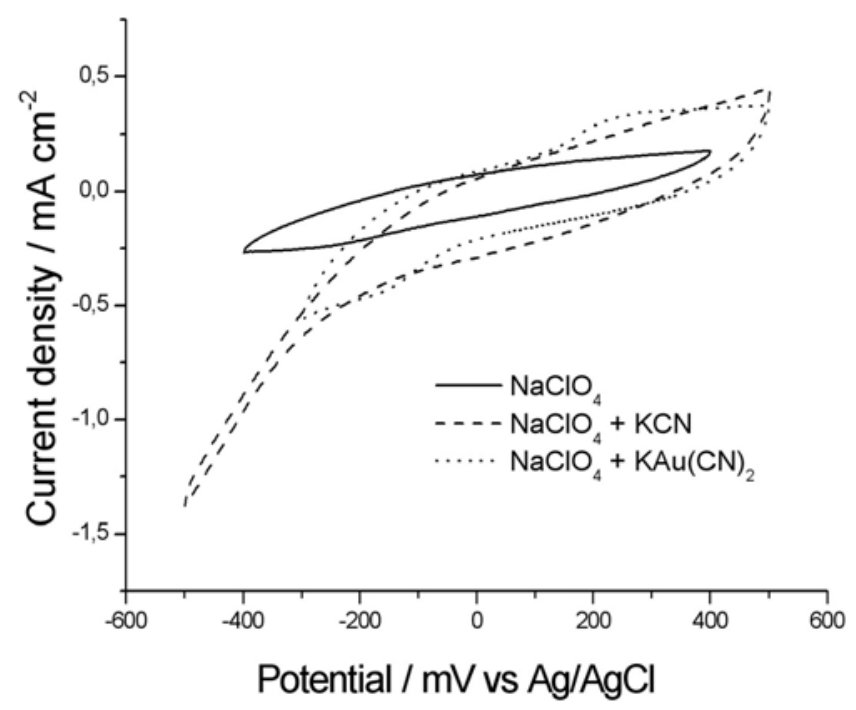

Fig. (1). Cyclic voltammetries of a polycrystalline Au electrode in contact with: (i) $\mathrm{NaClO}_{4} 0.1 \mathrm{M}$, (ii) $\mathrm{NaClO}_{4} 0.1 \mathrm{M}$, KCN $25 \mathrm{mM}$, (iii) $\mathrm{NaClO}_{4} 0.1 \mathrm{M}, \mathrm{KAu}(\mathrm{CN})_{2} 25 \mathrm{mM}$ solutions. Scan rate: $50 \mathrm{mV}$ $\mathrm{s}^{-1}$.

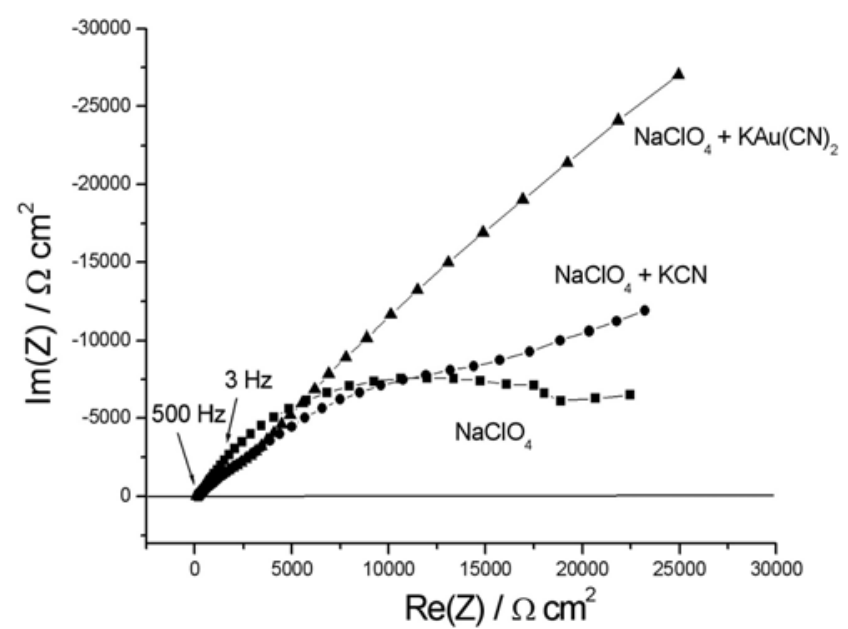

Fig. (2). Electrochemical impedance spectra, measured at $200 \mathrm{mV}$ vs $\mathrm{Ag} / \mathrm{AgCl}$, of polycrystalline $\mathrm{Au}$ in contact with: (i) $\mathrm{NaClO}_{4} 0.1$ M, (ii) $\mathrm{NaClO}_{4} 0.1 \mathrm{M}, \mathrm{KCN} 25 \mathrm{mM}$, (iii) $\mathrm{NaClO}_{4} 0.1 \mathrm{M}$, $\mathrm{KAu}(\mathrm{CN})_{2} 25 \mathrm{mM}$ solutions. Spanned frequency range: $65000 \mathrm{~Hz}$ to $2.5 \mathrm{mHz}$, potential modulation: $200 \mathrm{mV}$.

In Fig. (2) we report the Nyquist diagrams of EIS measurements carried out with the three systems; these experiments were performed in the frequency range from 65000 $\mathrm{Hz}$ to $2.5 \mathrm{mHz}$ - wider than that relevant to ERS measurements, i.e. $3 \div 500 \mathrm{~Hz}$ - with a sinusoidal potential modulation whose bias and amplitude correspond to the conditions employed in ERS investigations, i.e. bias: $200 \mathrm{mV}$, peak-topeak amplitude $200 \mathrm{mV}$. Of course, non-linear effects are 
present in these EIS spectra, but - in cases in which quantitative modelling work is required - these can be readily accounted for [13]; nevertheless, in the present case, only qualitative use is made of our EIS data. The spectral shape is dominated by mass-transport controlled $\mathrm{O}_{2}$ reduction; nevertheless the dynamic response is clearly influenced by the different electrolyte compositions. In particular, in the whole investigated frequency range, three intervals are found in which different interfacial relaxation mechanisms dominate. An insightful discussion of the relevant details is beyond the scope of the present paper, in this context, we simply stress that, in the frequency range accessible to ERS measurements a consistent electrochemical behaviour is found for the three systems, with a frequency response dominated by a single mechanism.

\subsection{Dynamic Response of Current Density}

In order to check whether the optical dynamics is dominated by the c.d. response (i.e. the purely electrical dynamics of the interface) or the two dynamic behaviours can be separated, we have measured the c.d. transients corresponding to the imposition of potential square-wave modulations between 0 and $400 \mathrm{mV}$, with frequencies in the interval $1 \div 300$ Hz. Two typical data sets are shown in Figs. (3 and 4), corresponding to square-waves of 5 and $75 \mathrm{~Hz}$, respectively. One can notice an essentially capacitive c.d. relaxation, as expected for an electrode in the DLC region; of course, the actual capacitance values depend on the electrode area.

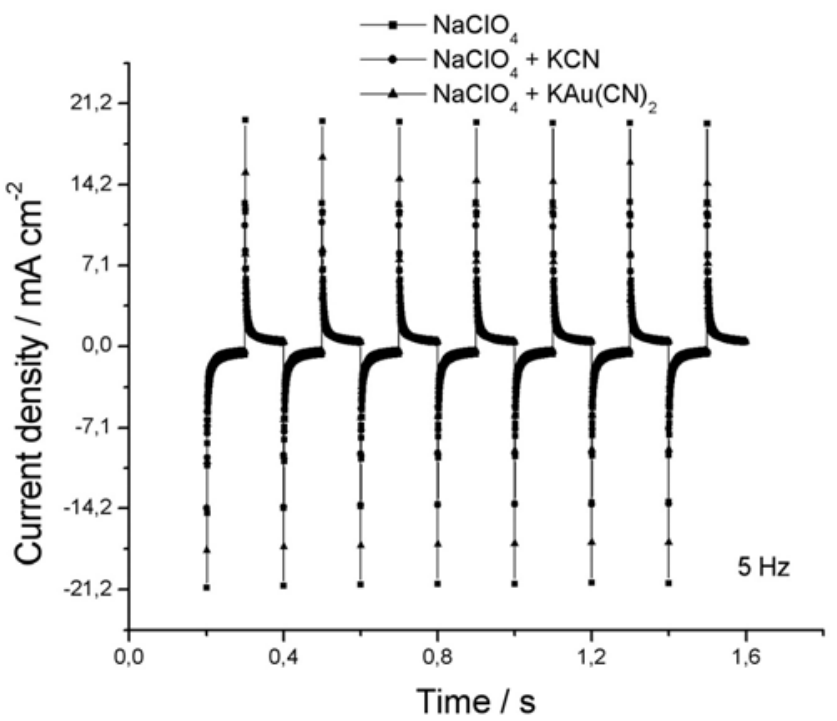

Fig. (3). Current density transients recorded during the application of potential square wave modulations between 0 and $400 \mathrm{mV}$ vs $\mathrm{Ag} / \mathrm{AgCl}$ with frequency of $5 \mathrm{~Hz}$. Working electrode: polycrystalline Au. Solutions: (i) $\mathrm{NaClO}_{4} 0.1 \mathrm{M}$, (ii) $\mathrm{NaClO}_{4} 0.1 \mathrm{M}, \mathrm{KCN} 25$ $\mathrm{mM}$, (iii) $\mathrm{NaClO}_{4} 0.1 \mathrm{M}, \mathrm{KAu}(\mathrm{CN})_{2} 25 \mathrm{mM}$.

In order to represent in a compact way the c.d. dynamic response, we computed the average c.d. variations recorded between the two potential levels:

$\Delta i=\frac{1}{T_{1}} \int_{0}^{T_{1}} i(t) d t-\frac{1}{T_{2}} \int_{0}^{T_{2}} i(t) d t$

Equation (1)

where $T_{1}$ and $T_{2}$ are the durations of the square-waves at 0 and $400 \mathrm{mV}$, respectively and $i$ represents the c.d.. In this case $T_{1}=T_{2}$. The reason for choosing this specific form of

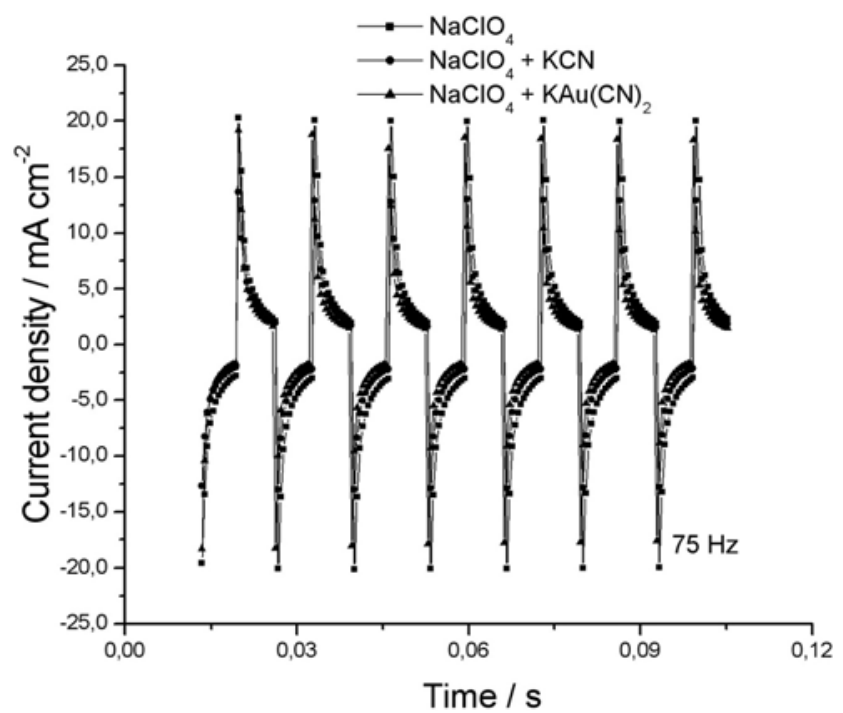

Fig. (4). Current density transients recorded during the application of potential square wave modulations between 0 and $400 \mathrm{mV}$ vs $\mathrm{Ag} / \mathrm{AgCl}$ with frequency of $75 \mathrm{~Hz}$. Working electrode: polycrystalline Au. Solutions: (i) $\mathrm{NaClO}_{4} 0.1 \mathrm{M}$, (ii) $\mathrm{NaClO}_{4} 0.1 \mathrm{M}, \mathrm{KCN} 25$ $\mathrm{mM}$, (iii) $\mathrm{NaClO}_{4} 0.1 \mathrm{M}, \mathrm{KAu}(\mathrm{CN})_{2} 25 \mathrm{mM}$.

Equation (1) will be evident in Section 3.3. The frequency dependence of $\Delta i(\omega)$ for the three systems investigated is shown in Fig. (5). From this Figure a clear difference in the dynamic behaviour of the three electrolytes is found. The growing trend with frequency measured in the relevant frequency range - corresponding to the presence of an overshoot in the c.d. transients measured after the switching of the potential between the two levels of interest - can be explained with the fact that electroreduction of $\mathrm{O}_{2}$ is taking place, giving rise to a background cathodic c.d.; this behaviour is coherent with the EIS spectral patterns, shown in Fig. (2) and discussed in Section 3.1. The dynamics of a DLC process is expected to exhibit a low-pass behaviour, implying a decrease of $\Delta i$ with $\omega$. Correspondingly, the c.d. tran-

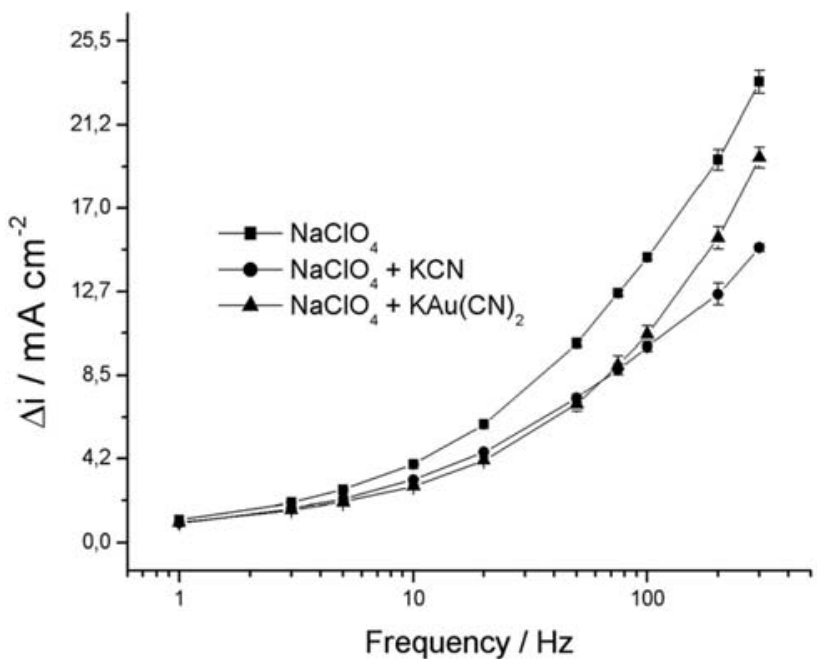

Fig. (5). Average current densities as a function of frequency, recorded during the application of potential square wave modulations between 0 and $400 \mathrm{mV}$ vs $\mathrm{Ag} / \mathrm{AgCl}$. Working electrode: polycrystalline Au. Solutions: (i) $\mathrm{NaClO}_{4} 0.1 \mathrm{M}$, (ii) $\mathrm{NaClO}_{4} 0.1 \mathrm{M}, \mathrm{KCN}$ $25 \mathrm{mM}$, (iii) $\mathrm{NaClO}_{4} 0.1 \mathrm{M}, \mathrm{KAu}(\mathrm{CN})_{2} 25 \mathrm{mM}$. 
Table 1. Time-Constants for the three Investigated Systems and their Respective 95\% Confidence Intervals, Estimated by NLLS Fitting of the Data Shown in Fig. (5) with Equation 2 (c.d. Transients) and in Figs. (6-10) with Equation 4 (Optical Response)

\begin{tabular}{|c|c|c|c|c|c|}
\hline & $\lambda$ & Potential modulation & $\mathrm{NaClO4}$ & $\mathrm{NaClO} 4+\mathrm{KCN}$ & $\mathrm{Au}(\mathrm{I})$ \\
\hline$\Delta i(\omega)$ & & $0 \div 400 \mathrm{mV}$ & $3.80 \pm 0.03$ & $7.80 \pm 0.15$ & $0.81 \pm 0,01$ \\
\hline \multirow{2}{*}{$\frac{\Delta R}{R}(\omega)$} & 440 & $0 \div 400 \mathrm{mV}$ & $14.60 \pm 9.15$ & $115.95 \pm 28.84$ & $14.15 \pm 5.80$ \\
\hline & 500 & $0 \div 400 \mathrm{mV}$ & $20.90 \pm 3.79$ & $200.28 \pm 77.14$ & $74.33 \pm 24.84$ \\
\hline
\end{tabular}

sients would exhibit a first-order relaxation behaviour, without an overshoot. One is thus led to interpret Fig. (5) as just the low-frequency portion of a more general $\Delta i(\omega)$ behaviour, implying the passage through a maximum and eventually a decrease, as $\omega$ is increased. The investigation of higher- $\omega$ behaviour is not of interest for our present research - where the sampling time is larger than that required to reach the maximum of the c.d. overshoot - since here we simply mean to compare the c.d. and optical dynamics in the $\omega$-range relevant to ERS measurements.

A simple model of this dynamic behaviour can be obtained by fitting the data of Fig. (5) with a first-order RC high-pass filter (Equation (2)).

$$
\Delta i(\omega)=\frac{\omega \tau_{i}}{\sqrt{1+\omega^{2} \tau_{i}^{2}}}
$$

The time-constants for the three systems and their respective $95 \%$ confidence intervals have been estimated by NLLS and are reported in Table $\mathbf{1}$.

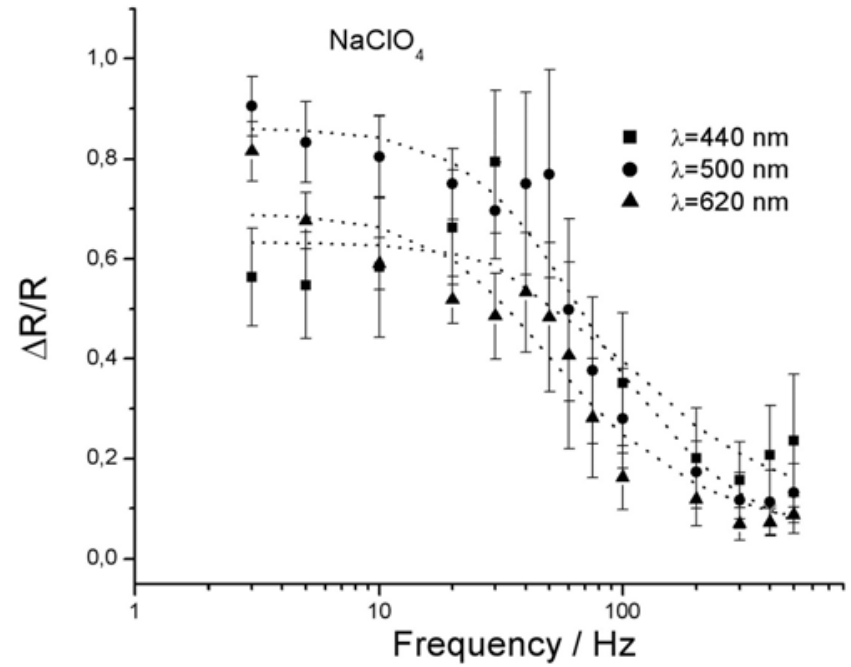

Fig. (6). Normalized change in reflectivity, $\Delta R / R$, as a function of frequency, recorded during the application of potential square wave modulations between 0 and $400 \mathrm{mV}$ vs $\mathrm{Ag} / \mathrm{AgCl}$. Working electrode: polycrystalline $\mathrm{Au}$. Solution: $\mathrm{NaClO}_{4} \mathrm{O} .1 \mathrm{M}$.

\subsection{Dynamics of the Optical Response}

We carried out ERS measurements for the three systems described in Section 2 by imposing potential square-waves between 0 and $400 \mathrm{mV}$ with frequencies in the range $3 \div 500$
$\mathrm{Hz}$. The region close to $50 \mathrm{~Hz}$ was not sampled, because in this range, the dynamic response is distorted by the presence of a notch filter, implemented in the hardware of our spectrometer. The ERS measurements were carried out at three visible wavelengths $\lambda$ : 440, 500 and $620 \mathrm{~nm}$, corresponding to spectral regions of low, intermediate (interband transition range) and high reflectivity for Au. Our results, expressed in terms of $\Delta R / R$ vs. $\omega$, are shown in Figs. (6-8). A decreasing trend is found, at variance with that of $\Delta i(\omega)$. This result seems to suggest that the $\Delta i(\omega)$ and $[\Delta R / R](\omega)$ dynamics are decoupled and that $[\Delta R / R](\omega)$ is not measurably affected by the $\mathrm{O}_{2}$ reduction reaction. $\Delta \mathrm{R} / \mathrm{R}$ is evaluated by electronic circuitry, implementing the algorithm:

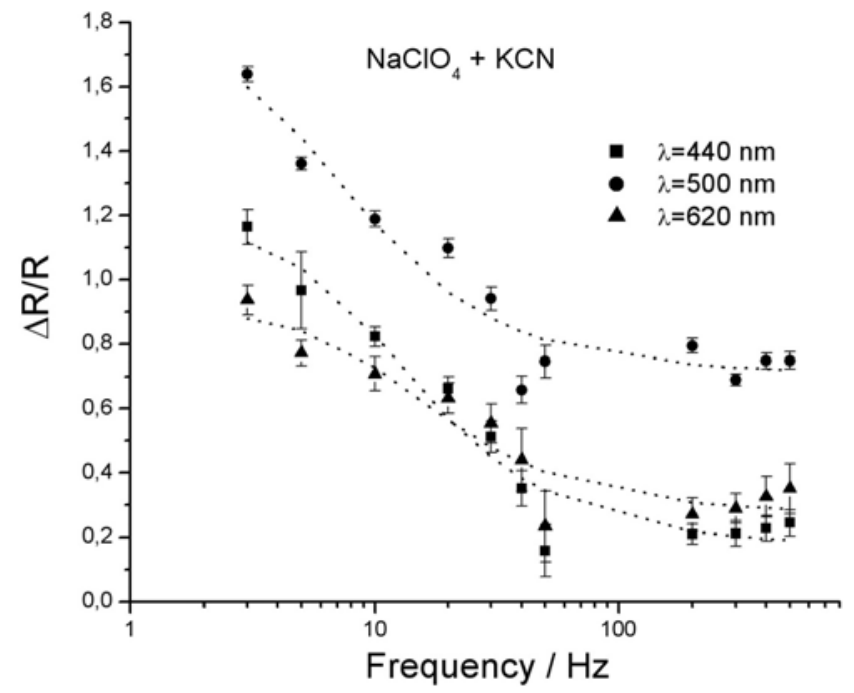

Fig. (7). Normalized change in reflectivity, $\Delta R / R$, as a function of frequency, recorded during the application of potential square wave modulations between 0 and $400 \mathrm{mV}$ vs $\mathrm{Ag} / \mathrm{AgCl}$. Working electrode: polycrystalline $\mathrm{Au}$. Solution: $\mathrm{NaClO}_{4} 0.1 \mathrm{M}, \mathrm{KCN} 25 \mathrm{mM}$.

$\frac{\Delta R}{R}=\frac{\frac{1}{T_{1}} \int_{0}^{T_{1}} R(t) d t-\frac{1}{T_{2}} \int_{0}^{T_{2}} R(t) d t}{\frac{1}{T_{1}+T_{2}} \int_{0}^{T_{1}+T_{2}} R(t) d t}$

Equation (3)

In our measurements: $\mathrm{T}_{1}=\mathrm{T}_{2}$. From Equation (3), the algorithmic choice leading to Equation (1) for the c.d. dynamic response should be clear: in the special case investigated $\frac{1}{T_{1}+T_{2}} \int_{0}^{T_{1}+T_{2}} R(t) d t \cong 0$ and the normalisation with this quan- 
tity would lead to obvious numerical problems. In Figs. (6-8) one can notice a systematic decreasing trend of $\Delta R / R$ with $\omega$. Furthermore, it is possible to observe differences in the details of the dynamic response of the three electrolytes as well as effects of the radiation wavelength $\lambda$ employed. In particular, one can notice that, with the $\mathrm{NaClO}_{4}$ solution, no significant changes in $[\Delta R / R](\omega)$ are caused by changing $\lambda$. In the presence of $\mathrm{CN}^{-}$(i.e. with $\mathrm{KCN}-$ and $\mathrm{KAu}(\mathrm{CN})_{2}-$ containing solutions) the $[\Delta R / R](\omega)$ curves are higher when $\lambda=500 \mathrm{~nm}$. This result can be explained by the fact that the interband transition energy range is more sensitive to modifications of surface electronic structure, brought about by the adsorption of the pseudohalogenide. In addition to the experiments carried out in the DLC region, for the $\mathrm{CN}^{-}$containing systems, we also carried out ERS measurements with the anodic voltage step set at such a potential that $\mathrm{Au}$ tends to corrode, giving rise to the formation of highly water-soluble $\mathrm{Au}(\mathrm{CN})_{2}{ }^{-}$([14] and references therein contained): the corresponding results are shown in Figs. ( 9 and 10). The trend of $[\Delta R / R](\omega)$ does not change, but appreciable differences are found in the absolute values and details of the frequency behaviour. A simple model was sought of the $\omega$-dependence shown in Figs. (6-10), exhibiting an obvious anti-correlation between $\Delta \mathrm{R} / \mathrm{R}$ and $\omega$, as well as a sigmoidal behaviour. We chose a first-order RC low-pass filter (Equation (4)) as a tentative analytic representation of these dynamics:

$\frac{\Delta R}{R}(\omega)=\frac{A}{\sqrt{1+\omega^{2} \tau_{o}^{2}}}+B$

Equation (4)

We evaluated the optical time-constants $\tau_{\mathrm{o}}$ and their $95 \%$ confidence intervals by NLLS fitting (the fitting curves are reported in Figs. (6-8)); the results are reported in Table $\mathbf{1 .}$

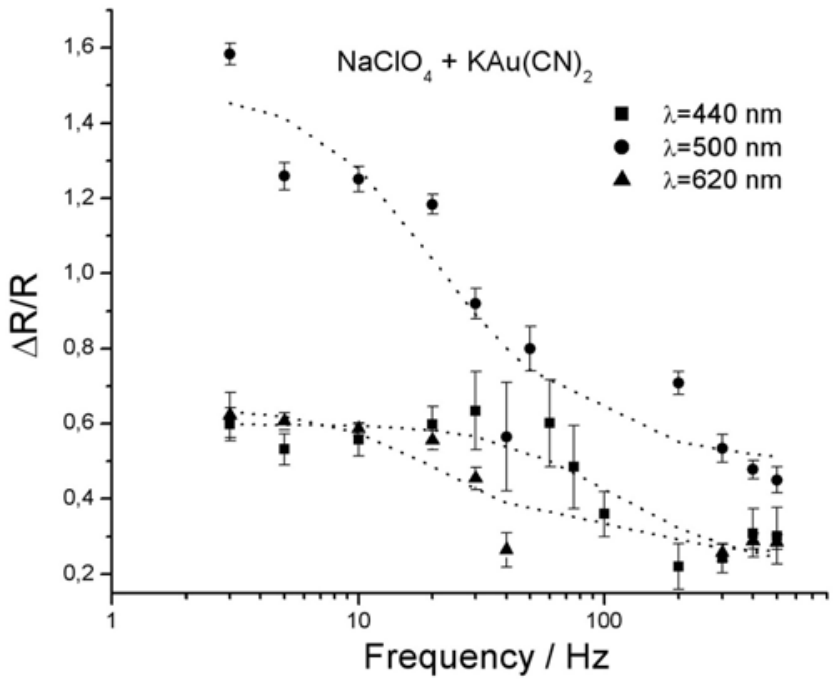

Fig. (8). Normalized change in reflectivity, $\Delta R / R$, as a function of frequency, recorded during the application of potential square wave modulations between 0 and $400 \mathrm{mV}$ vs $\mathrm{Ag} / \mathrm{AgCl}$. Working electrode: polycrystalline Au. Solution: $\mathrm{NaClO}_{4} 0.1 \mathrm{M}, \mathrm{KAu}(\mathrm{CN})_{2} 25$ $\mathrm{mM}$.

The differences among solutions and radiation wavelengths, as well as in anodic level, can be accurately fol- lowed with the optical time constant $\tau_{\mathrm{o}}$. In particular, it is worth observing that the $\tau_{\mathrm{o}}$ values for the $\mathrm{CN}^{-}$-containing systems are considerably larger if the anodic level of the potential square-wave exceeds the corrosion threshold, coherently with the well-known presence of hysteretic phenomena related to $\mathrm{Au}$ corrosion processes [15-17].

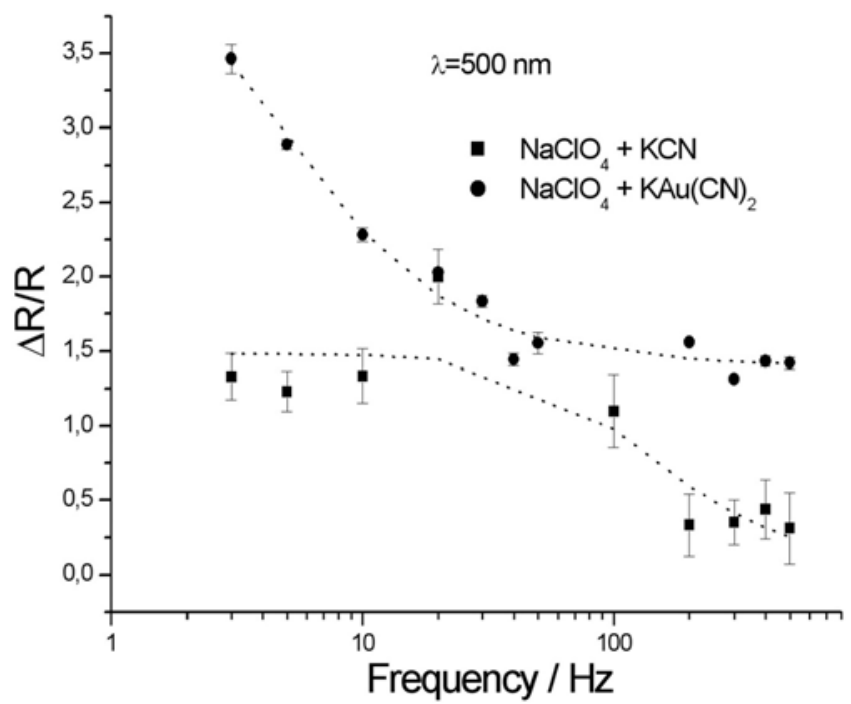

Fig. (9). Normalized change in reflectivity, $\Delta R / R$, as a function of frequency, recorded during the application of potential square wave modulations between 0 and $700 \mathrm{mV}$ vs $\mathrm{Ag} / \mathrm{AgCl}$. Wavelenght: 500 nm. Working electrode: polycrystalline Au. Solutions: (i) $\mathrm{NaClO}_{4}$ $0.1 \mathrm{M}, \mathrm{KCN} 25 \mathrm{mM}$, (ii) $\mathrm{NaClO}_{4} 0.1 \mathrm{M}, \mathrm{KAu}(\mathrm{CN})_{2} 25 \mathrm{mM}$.

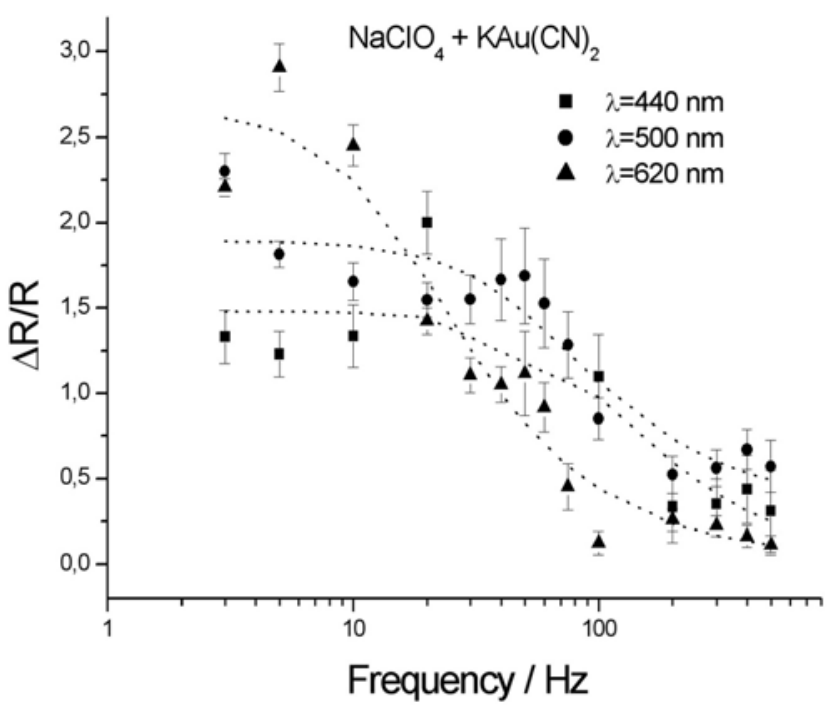

Fig. (10). Normalized change in reflectivity, $\Delta R / R$, as a function of frequency, recorded during the application of potential square wave modulations between 0 and $700 \mathrm{mV}$ vs $\mathrm{Ag} / \mathrm{AgCl}$. Working electrode: polycrystalline $\mathrm{Au}$. Solution: $\mathrm{NaClO}_{4} 0.1 \mathrm{M}, \mathrm{KAu}(\mathrm{CN})_{2} 25$ $\mathrm{mM}$.

Our results show that electroreflectance spectroscopy can be used also in a frequency-dependent mode, thus allowing to perform new measurements of the optical impedance of the electrochemical interface. The sensitivity of the "optical impedance" to the details of the interfacial electronic structure is liable to yield original information on electrodeposition and corrosion mechanisms as well as on the action of 
adsorbed additives and inhibitors. In [6] a firstapproximation version of a theory describing the changes in optical properties brought about by the electrodeposition process was proposed and in [7] an analytical expression was derived for the frequency dependence of the potentialmodulated UV-visible reflectance spectroscopic signal for redox reactions.

\section{CONCLUSIONS}

The qualitative comparison of current-density $\Delta i(\omega)$ and optical $[\Delta R / R](\omega)$ responses to potential square-waves of frequency $\omega$ shows opposite trends, in the frequency range studied. From a more quantitative point of view, it is found that the current-density and optical time constants $\tau_{\mathrm{i}}$ and $\tau_{\mathrm{o}}$ are well separated for all the systems investigated, notwithstanding the differences in electrolyte chemistry and radiation wavelength employed: in particular $\tau_{\mathrm{i}}<<\tau_{\mathrm{o}}$. Since the rise-time of the electronics is less than $1 \mu \mathrm{s}$, the instrumental response is over three orders of magnitude faster than the fastest processes studied in this study. It can thus be reliably concluded that the current-density and optical dynamics considered in the present investigation are: (i) operating on different time-scales, (ii) sensitive to the electrolyte chemistry and (iii) responsive to the radiation-wavelength. The latest property allows interfacial electronic structure information to be extracted from $[\Delta \mathrm{R} / \mathrm{R}](\omega)$ measurements.

\section{ACKNOWLEDGMENTS}

Collaboration with data analysis is gratefully acknowledged to Mr. Stefano Maci. The authors are also indebted to
Mr. Francesco Bogani for his high expertise in carrying out many electrochemical measurements.

\section{REFERENCES}

[1] Kolb, D.M. In Spectroelectrochemistry - Theory and Practice; Gale, R.J. Ed.; New York, NJ 1988; pp. 87-188.

[2] Kolb, D.M. In Advances in Electrochemistry and Electrochemical Engineering; Gerischer, H.; Tobias C.W. Eds.; J. Wiley \& Sons, NY 1978; Vol. 11, p. 125.

[3] McIntyre, J.D.E.; Aspnes, D.E. Surf. Sci. 1971, 24, 417.

[4] Takamura, T.; Takamura, K.; Nippe, W.; Yeager, E. J. Electrochem. Soc. 1970, 117, 626.

[5] Bewick, A.; Thomas, B. J. Electroanal. Chem. 1975, 65, 911.

[6] Bozzini, B.; Mele, C.; D'Urzo, L. J. Appl. Electrochem. 2006, 36, 87.

[7] Sagara, T.; Murase, H.; Nakashima, N. J. Electroanal. Chem. 1998, 454,75

[8] Tucceri, R.; Surface Science Reports 2004, 56, 85

[9] Anderson, W.J.; Hansen, W.N. J. Electroanal. Chem. 1973, 43, 329

[10] Hansen, W.N. J. Electroanal. Chem. 1973, 47, 229

[11] Tucceri, R.; Posadas, D. J. Electroanal Chem. 1982, 131, 377

[12] Valinchius, G. Russ. J. Elecrochem. 1995, 31, 81

[13] Sgura, I.; Bozzini, B. Int. J. Non-Linear Mechanics 2005, 40, 557.

[14] Bozzini, B.; Mele, C.; Romanello, V. J. Electroanal. Chem. 2006, $592,25$.

[15] Kozin, L. F.; Prokopenko, V. A.; Bogdanova, A. K. Prot. Met., 2005, Vol. 41 No. 1, 22.

[16] Bozzini, B.; Giovannelli, G.; Natali, S.; Fanigliulo, A.; Cavallotti, P.L. J. Mater. Sci. 2002, 37, 3903.

[17] Bozzini, B.; Mele, C.; Romanello, V. Surf. Coatings Technol. 2007, $201,6267$.
Received: September 04, 2007

Revised: December 06, 2007

Accepted: December 07, 2007 